\title{
Anti-Nogo-A Antibodies As a Potential Causal Therapy for Lower Urinary Tract Dysfunction after Spinal Cord Injury
}

\author{
Marc P. Schneider, ${ }^{1,2,3 *}$ Andrea M. Sartori, ${ }^{1,2,3 *}$ Benjamin V. Ineichen, ${ }^{1,2}$ Selina Moors, ${ }^{1,2}$ Anne K. Engmann, ${ }^{1,2}$ \\ Anna-Sophie Hofer, ${ }^{1,2}$ Oliver Weinmann, ${ }^{1,2}$ Thomas M. Kessler, ${ }^{3}$ and Martin E. Schwab ${ }^{1,2}$ \\ ${ }^{1}$ Brain Research Institute, University of Zürich, ${ }^{2}$ Department of Health Sciences and Technology, Eidgenössiche Technische Hochschule Zürich, 8057 \\ Zürich, Switzerland, and ${ }^{3}$ Department of Neuro-Urology, Balgrist University Hospital, University of Zürich, 8008 Zürich, Switzerland
}

Loss of bladder control is common after spinal cord injury (SCI) and no causal therapies are available. Here we investigated whether function-blocking antibodies against the nerve-fiber growth inhibitory protein Nogo-A applied to rats with severe SCI could prevent development of neurogenic lower urinary tract dysfunction. Bladder function of rats with SCI was repeatedly assessed by urodynamic examination in fully awake animals. Four weeks after SCI, detrusor sphincter dyssynergia had developed in all untreated or control antibody-infused animals. In contrast, 2 weeks of intrathecal anti-Nogo-A antibody treatment led to significantly reduced aberrant maximum detrusor pressure during voiding and a reduction of the abnormal EMG high-frequency activity in the external urethral sphincter. Anatomically, we found higher densities of fibers originating from the pontine micturition center in the lumbosacral gray matter in the anti-Nogo-A antibody-treated animals, as well as a reduced number of inhibitory interneurons in lamina X. These results suggest that anti-Nogo-A therapy could also have positive effects on bladder function clinically.

Key words: CRF; neuro-urology; neurogenic lower urinary tract dysfunction; Nogo-A; spinal cord injury; urodynamics

Significance Statement

After spinal cord injury, loss of bladder control is common. Detrusor sphincter dyssynergia is a potentially life-threatening consequence. Currently, only symptomatic treatment options are available. First causal treatment options are urgently needed in humans. In this work, we show that function-blocking antibodies against the nerve-fiber growth inhibitory protein Nogo-A applied to rats with severe spinal cord injury could prevent development of neurogenic lower urinary tract dysfunction, in particular detrusor sphincter dyssynergia. Anti-Nogo-A therapy has entered phase II clinical trial in humans and might therefore soon be the first causal treatment option for neurogenic lower urinary tract dysfunction.

\section{Introduction}

Many neurological diseases that affect descending tract systems, including spinal cord injury (SCI) and multiple sclerosis, often

Received Dec. 17, 2018; accepted Feb. 6, 2019.

Author contributions: M.P.S., A.M.S., A.K.E., T.M.K., and M.E.S. designed research; M.P.S., A.M.S., B.V.I., S.M., A.K.E., A.-S.H., O.W., T.M.K., and M.E.S. performed research; M.P.S., A.K.E., T.M.K., and M.E.S. contributed unpublished reagents/analytic tools; M.P.S., A.M.S., 0.W., and M.E.S. analyzed data; M.P.S. and M.E.S. wrote the paper.

This work was supported by grants from the Swiss Continence Foundation, the Swiss National Science Foundation, the European Research Council (Advanced Grant Nogorise to M.E.S.), the Christopher and Dana Reeve Foundation, and the Santa Casa da Misericórdia de Lisboa (Prémio Mello e Castro - 2016). This work was awarded the Swiss Continence Foundation Award at the 5th International Neuro-Urology Meeting, 25 -28 January 2017, Zürich, Switzerland. We thank Prof. P. Sawchenko and Dr. W. Vale from the Salk Institute for Biological Studies for giving us access to their CRF antibody. We thank Pietro Morciano and Frank David from the information technology section of the Brain Research Institute, University of Zürich, as well as Stefan Giger, Hansjörg Kasper, Marco Tedaldi, and Martin Wieckhorst from the infrastructure team of the Brain Research Institute, University of Zürich, for their support.

*M.P.S. and A.M.S. contributed equally to this work.

None of the funding organizations had a role in the design and conduct of the study, collection, management, analysis, and interpretation of the data, preparation, review, or approval of the manuscript, or in the decision to submit the manuscript for publication. M.E.S. is the cofounder and president of the board of NovaGo Therapeutics Inc. lead to severe disturbances of bladder function. Detrusor sphincter dyssynergia (DSD; Weld et al., 2000), which is defined by dyssynergic contractions of the external urethral sphincter (EUS) during the voiding phase, is a life-threatening complication because it results in high intravesical pressure with subsequent urine reflux to the kidneys. Over years, this can lead to kidney damage and eventually renal failure (Groen et al., 2016). Lower urinary tract dysfunction contributes in a major way to a decreased quality of life in the affected patients and therefore is one of the highest rehabilitation priorities (Simpson et al., 2012). However, only a few symptomatic treatment options are currently available, including intermittent self-catheterization four to six times a day, antimuscarinic drugs, and intradetrusor

\section{Correspondence should be addressed to Marc P. Schneider at mpschneider@outlook.com.}

M.P. Schneider's present address: Department of Urology, Bern University Hospital, Inselspital, University of Bern, 3010 Bern, Switzerland.

https://doi.org/10.1523/JNEUROSCI.3155-18.2019

Copyright $\odot 2019$ the authors 
onabotulinumtoxin-A injections (Panicker et al., 2015). These treatments are at best symptomatic and come at the cost of frequent side effects, such as urinary tract infections, or injuries to the urethra. Causal treatment options for neurogenic lower urinary tract dysfunctions, in particular DSD, are therefore urgently needed (Panicker et al., 2015).

The central neuronal circuits controlling lower urinary tract function are not yet fully understood. Storage and voiding of the urine are achieved by complex interactions between the somatic and the autonomic nervous systems (de Groat et al., 2015). Although the storage reflex is mainly an intraspinal process (De Groat and Lalley, 1972), the initiation of voiding depends on supraspinal input from a nucleus located in the pons, the pontine micturition center (PMC; Griffiths et al., 1990). The PMC-spinal projection neurons, which are excitatory and glutamatergic, contain the neuropeptide corticotropin-releasing factor (CRF; Verstegen et al., 2017). These neurons send long-projecting axons to the lumbosacral cord and play a fundamental role in micturition. A lesion to the spinal cord disrupts the neural circuitry controlling the lower urinary tract function, and the neurourological consequences for the patient depend on the location and the extent of the injury. After a suprasacral SCI, the voluntary, supraspinal control of voiding is interrupted, leading to an initial acontractile bladder, causing urinary retention. After weeks to months, depending on the species, autonomic voiding slowly reappears and culminates in detrusor overactivity (de Groat et al., 1990), which was hypothesized to be the result of aberrant intraspinal plasticity with an important contribution by C-fibers (Cheng and de Groat, 2004). Although bladder contraction reappears over time, voiding is usually inefficient due to the simultaneous contraction of the EUS together with the detrusor, a phenomenon that is known as detrusor-sphincter DSD. DSD arises over time in the majority of patients with an incomplete or complete SCI, causing elevated intravesical pressures that, over time, put the upper urinary tract at risk (Bacsu et al., 2012; Groen et al., 2016).

Bladder function can be determined with high precision by urodynamic measurements, i.e., by simultaneously assessing the intravesical pressure and EUS electromyography under fully awake conditions (Nosseir et al., 2007). This clinical protocol has recently been transferred and reproduced in intact and spinal cord-injured rats (Schneider et al., 2015; Foditsch et al., 2018).

Myelin-enriched membrane protein Nogo-A, a potent nervefiber growth inhibitory protein, is partly responsible for the low level of spontaneous neuronal regeneration and repair in the adult mammalian CNS (Schwab, 2010; Schwab and Strittmatter, 2014). Neutralization by functionally blocking antibodies, genetic deletion of Nogo-A, or blockade of Nogo-A receptors induces substantial axonal regeneration, as well as enhanced neuronal plasticity and functional recovery after SCI or stroke in animal models (Liebscher et al., 2005; Schwab and Strittmatter, 2014; Wahl et al., 2014). It has been noted before that inhibition of Nogo-A in rats with large but incomplete SCIs using functionblocking antibodies not only resulted in improved recovery of locomotion, but also led to an earlier restoration of independent voiding (Liebscher et al., 2005). However, no one so far has conducted a detailed urodynamic assessment of anti-Nogo-A antibodies as a potential therapy for DSD following SCI. The present study investigates the effects of anti-Nogo-A antibodies in the development of neurogenic lower urinary tract dysfunction, in particular DSD, following a large SCI in adult rats.

\section{Materials and Methods}

Rats. Adult female Lewis rats [LEW/OrlRj (Lewis); weight, $210 \pm 20 \mathrm{~g}$; age, $4 \pm 1$ months] were purchased from Janvier. The rats were housed in groups of 3-4 per cage, and single-housed after catheter implantation. Food (rat chow) and water were provided ad libitum. Rats were maintained on a $12 \mathrm{~h}$ light/dark cycle (light on from 6:00 A.M. until 6:00 P.M.). All experiments were approved by the Veterinary Office of the canton of Zürich, Switzerland (license 19/2014) and followed approved guidelines and regulations.

Catheter and electrodes implantation. All rats underwent initial preoperative handling and acclimatization to the urodynamic laboratory station followed by catheter and electrode implantation. Procedures were performed as described previously (Schneider et al., 2015; Foditsch et al., 2018). Briefly, rats were initially anesthetized in 5\% isoflurane (Piramal Healthcare) in air and maintained by an intramuscular injection of medetomidine $(0.15 \mathrm{mg} / \mathrm{kg}$; Orion Pharma, Domitor), midazolanum (2 $\mathrm{mg} / \mathrm{kg}$; Roche, Dormicum), and fentanyl (5 $\mu \mathrm{g} / \mathrm{kg}$; Kantonsapotheke University Hospital Zurich). Bladder catheters were inserted into the bladder dome and secured with a purse-string suture. EMG electrodes were affixed to the fat tissue beside the EUS, and a ground electrode was sutured to the abdominal muscle. Catheter and wires were tunneled subcutaneously to the back of the neck, exteriorized, and attached to an infusion harness (SAI Infusion Technologies, QC Single).

SCI. Rats were anesthetized as described above. In 41 animals, the T8 lamina was removed and the dorsal two-thirds of the spinal cord were transected bilaterally microsurgically with iridectomy scissors, resulting in severe but incomplete SCI. In 28 rats, the lower thoracic spinal cord was transected completely. Seventeen rats were left intact.

Osmotic pump implantation. Fifty-four spinal cord injured rats were implanted with intrathecal pumps for antibody delivery at the day of SCI. Following baseline urodynamic measurements, the rats were divided into four different groups: incomplete SCI with control antibody treatment $(n=17)$, complete SCI with control antibody treatment $(n=10)$, incomplete SCI with anti-Nogo-A antibody treatment $(n=16)$, and complete SCI with anti-Nogo-A antibody treatment $(n=11)$. An a priori power calculation (power, $80 \% ; \alpha=0.05$ ) indicated a minimal group size of $n=10$ rats. The pump-implantation procedure was performed as previously described (Ineichen et al., 2017). Briefly, immediately following the SCI, a second laminectomy at vertebrate level L2 was performed and a fine intrathecal catheter ( 32 gauge; ReCathCo) was inserted into the subarachnoid space and pushed in cranial direction toward the lesion for $1 \mathrm{~cm}$. The catheter was connected to an osmotic minipump (Alzet, 2ML2), which continuously delivered over $14 \mathrm{~d}$ either an anti-Nogo-A antibody (monoclonal antibody 11C7; Oertle et al., 2003) or an inactive control antibody (anti-BrdU; Serotec) into the intrathecal space $(5 \mu \mathrm{l} / \mathrm{h}$, $3 \mathrm{mg}$ of antibody/ml). In this way, $6 \mathrm{mg}$ of antibodies were applied in total over $14 \mathrm{~d}$. Pump and catheter were removed 15-16 d after implantation under 5\% isoflurane (Piramal Healthcare) anesthesia, and the skin was closed by sutures.

Urodynamic and EUS-EMG measurements. Procedures were performed as previously described (Schneider et al., 2015; Foditsch et al., 2018). Acclimatization of the rats to the urodynamic setup was performed before catheter and electrode implantation. Awake rats were positioned in a modified restrainer (modified from item \#HLD-RM, Kent Scientific) with a hole situated under the orifice of the urethra. The restrainer was then placed in a modified Small Animal Cystometry Lab Station (Catamount Research and Development) with a scale below the funnel. The bladder catheter was attached to a syringe pump with an in-line pressure transducer, and the electrodes were connected to an amplifier/converter. Saline was instilled $(120 \mu \mathrm{l} / \mathrm{min})$, and all parameters (pressure, scale, voltage) recorded simultaneously for $\geq 3$ voiding cycles. Urodynamic measurements were performed during the light phase. For data assessment, the maximum voiding detrusor pressure was measured as the peak of the intravesical pressure during voiding (see Fig. 2a). Voided volume was measured as the change in weight on the scale (assuming a density approaching 1 ). To measure the rigidity of the bladder wall, bladder compliance was calculated as the intravesical pressure increase (threshold detrusor pressure minus baseline detrusor pressure) 
a

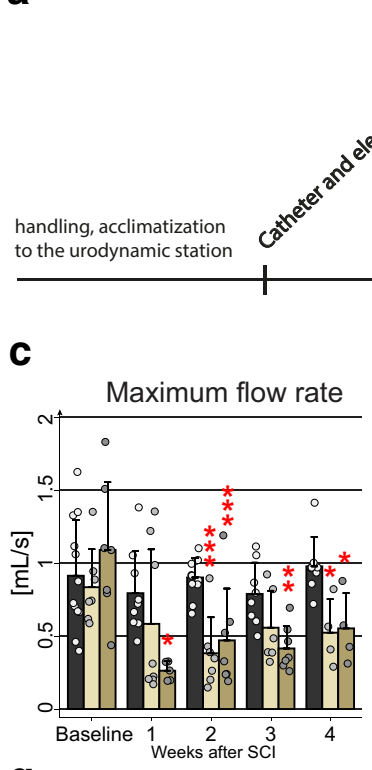

g

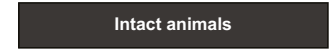

Occurrence of high frequency EMG activity
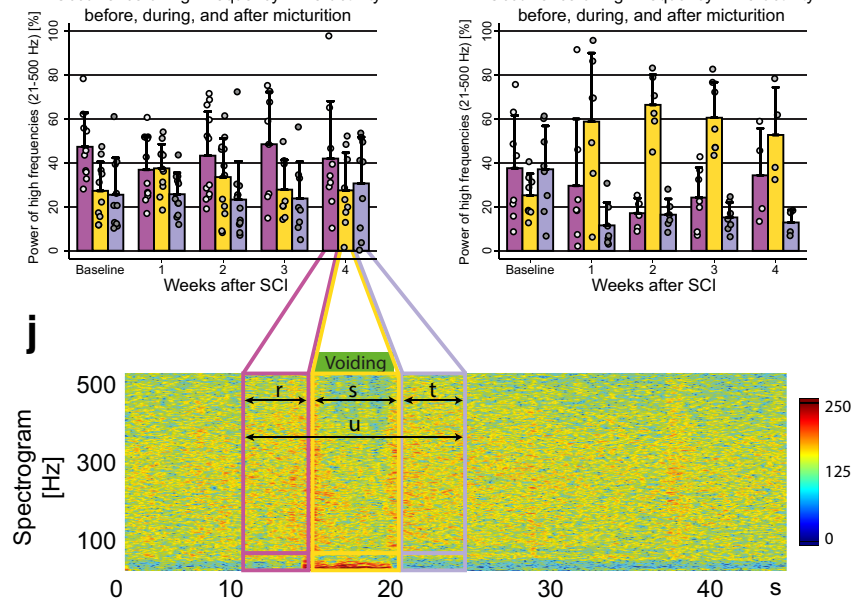

d

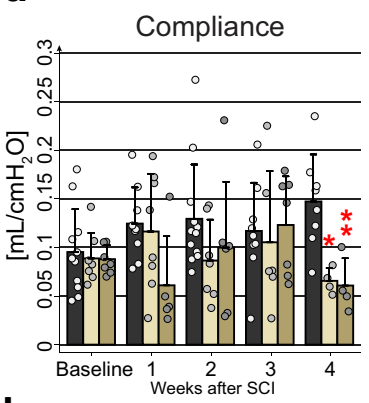

h

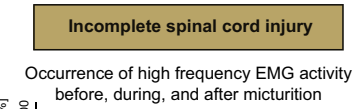

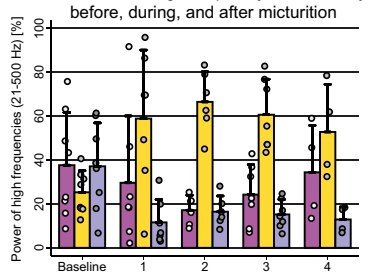

i
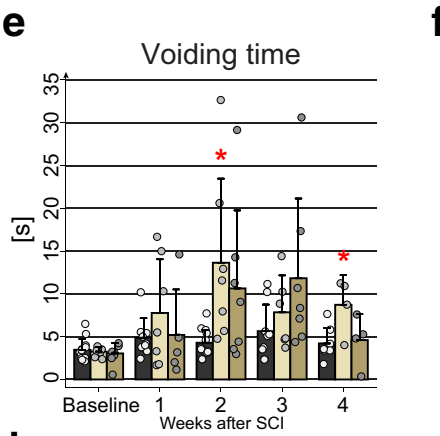

f

b
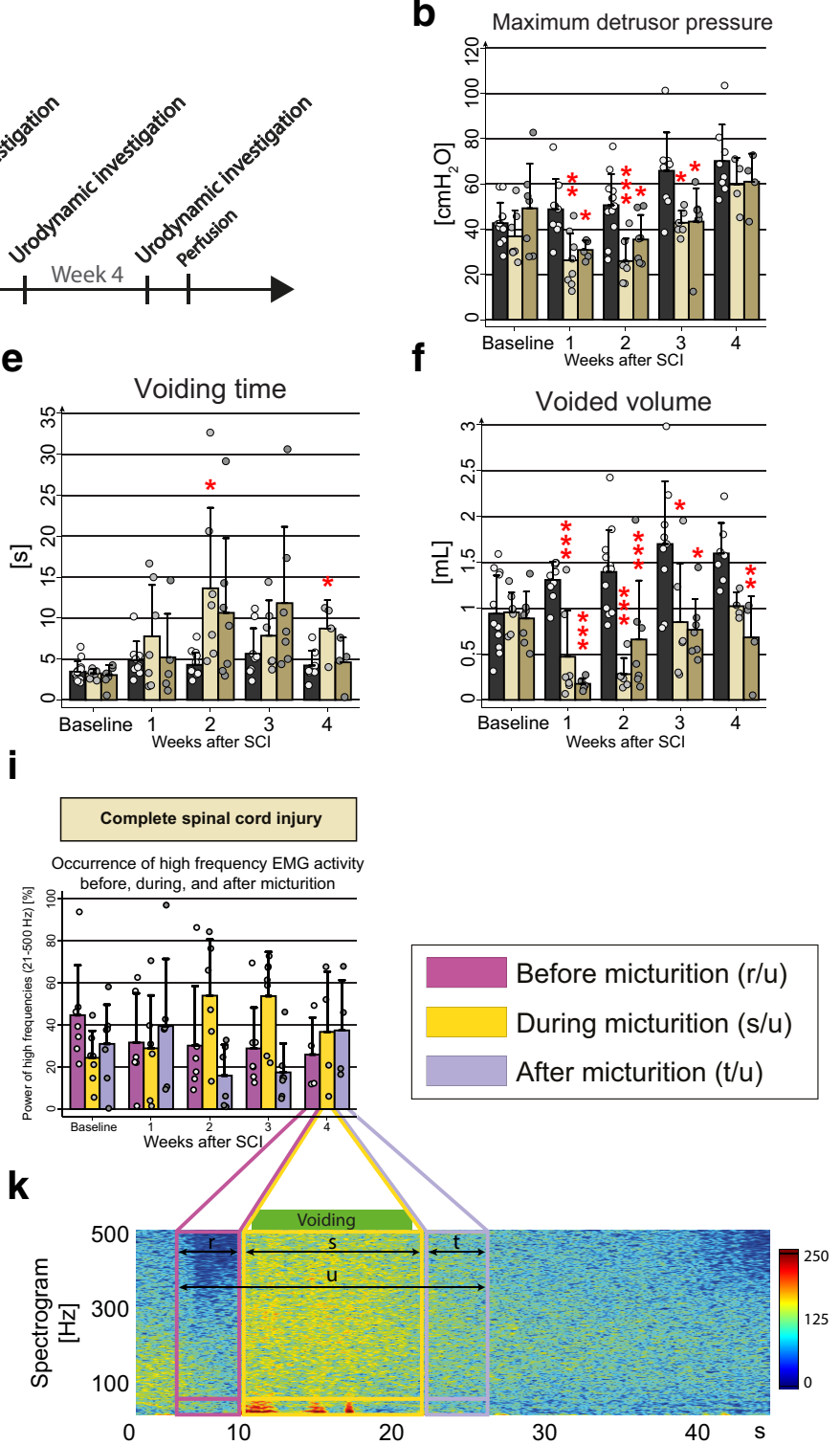

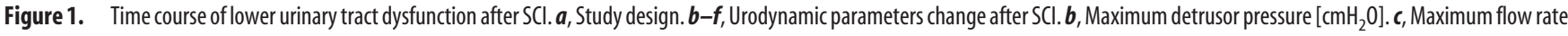
$[\mathrm{ml} / \mathrm{s}]$. $\boldsymbol{d}$, Bladder compliance [ml/ $\left.\mathrm{cmH}_{2} 0\right]$. $\boldsymbol{e}$, Voiding time [s]. $\boldsymbol{f}$, Voided volume [ml]. $\boldsymbol{g}$ - $\boldsymbol{k}$, EUS-EMG analysis before and 1, 2, 3, and 4 weeks after incomplete or complete SCl. $\boldsymbol{g}$, EMG power spectrum shows highest percentage power for the high frequencies $(21-500 \mathrm{~Hz})$ before voiding in intact rats. $\boldsymbol{h}$, After a large but incomplete $\mathrm{SCl}$, the high-frequency power activity $(21-500 \mathrm{~Hz})$ of the EUS shifts to the micturition phase starting at day 7, a condition typical for DSD. $i$, Similar but less pronounced shift in complete SCI rats. $j$, EUS-EMG spectrogram of one voiding cycle of an intact rat showing increased high-frequency power activity $(21-500 \mathrm{~Hz}$, bigger quadrant) before micturition, followed by slow-wave bursting activity (1-20 Hz, smaller quadrant) during micturition. $\boldsymbol{k}$, EUS-EMG spectrogram of one voiding cycle of an incompletely spinal cord-injured rat 4 weeks after $S C l$. Percentual power in $g$-i was calculated by dividing the power of high frequencies in the period of interest (before, $r$; during, $s$; after, t) by the whole analyzed period (u). Statistical testing: data of intact and both complete and incomplete $\mathrm{SCl}$ were analyzed with a one-way repeated-measures ANOVA followed by Bonferroni's post hoc testing; ${ }^{*} p<0.05 ;{ }^{* *} p<0.01$; ${ }^{* * *} p<0.001$.

divided by the voided volume. Urodynamic and behavioral assessments were performed on randomly number-coded animals in a fully blinded fashion. Measurements were analyzed blinded using an adapted LabVIEW program.

EMG analysis. The EUS-EMG was bandpass-filtered ( $2 \mathrm{~Hz}$ to $2 \mathrm{kHz}$ ). The frequency spectrogram was analyzed with a self-programmed application based on LabVIEW version 2012 (National Instruments). The 45-s-long EMG signal was sliced into 4096 samples, of which 3596 were overlapping (shift is 500 samples), creating a typical graphical representation of a spectral heatplot. After taking a Hanning window of the signal, a fast Fourier transformation was generated. As a result, we obtained a power value for each frequency at every computed time point. Before graphic processing, the signal was up-scaled by a multiplication factor to assure that the signal consists, after logarithmizing, mainly of positive values. The logarithmized signal was also scaled up by factor of 20 . This signal was then plotted as a color map with Jet Colormap. All frequencies $>500 \mathrm{~Hz}$ were neglected.

Quantification of high-frequency EUS-EMG activity was achieved by summing every high-frequency power value in the period of interest (Fig. $1 j, k$; before micturition, $\mathrm{r}$; during micturition, $\mathrm{s}$; after micturition, $\mathrm{t}$ ) and dividing it by the sum of every high-frequency power value in the whole period analyzed (Fig. $1 j, k, \mathrm{u})$. Data are shown in percentage.

Perfusion, fixation, and tissue preparation. Rats were killed with an intraperitoneal overdose of pentobarbital ( $300 \mathrm{mg} / \mathrm{ml}$; Streuli Pharma). Animals were transcardially perfused first with $150 \mathrm{ml}$ of Ringer solution (B. Brown Medical) containing 1\% heparin (B. Brown Medical), followed by $300 \mathrm{ml}$ of $4 \%$ paraformaldehyde solution (Sigma-Aldrich) containing $5 \%$ sucrose and phosphate-buffered at $\mathrm{pH}$ 7.4. Spinal cords were 
a
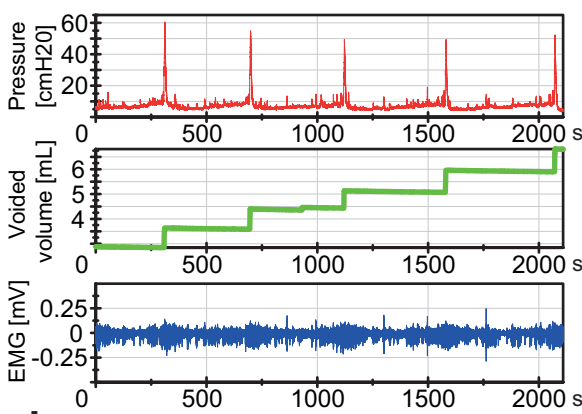

d
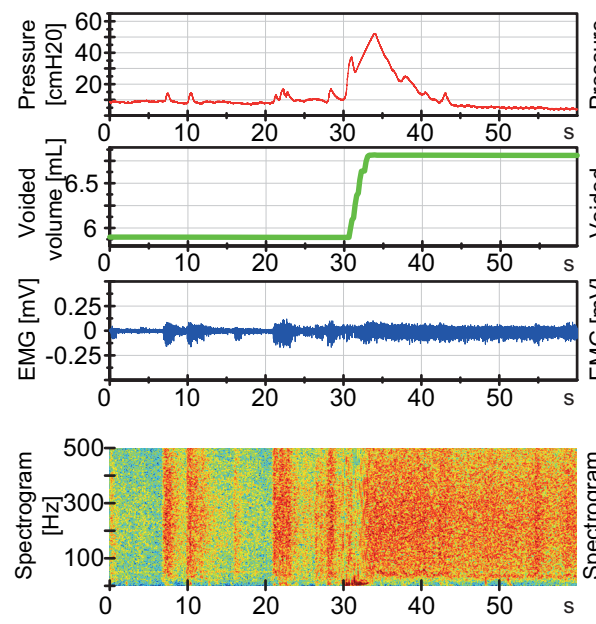

b
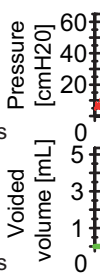

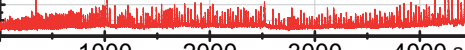

7 days after injury

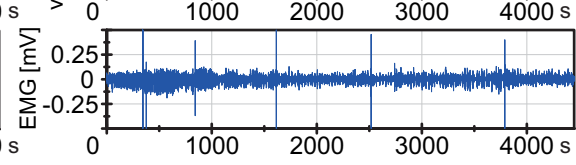

e
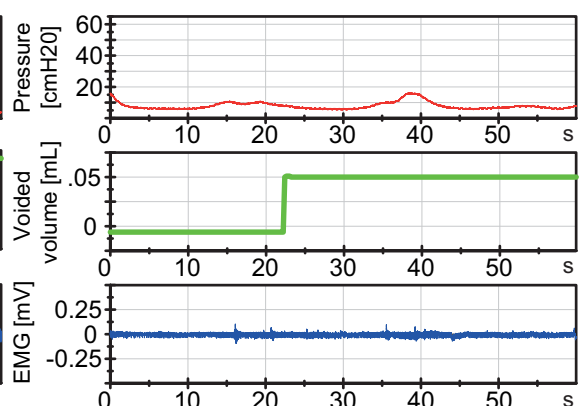

C

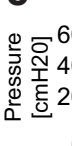

28 days after injury

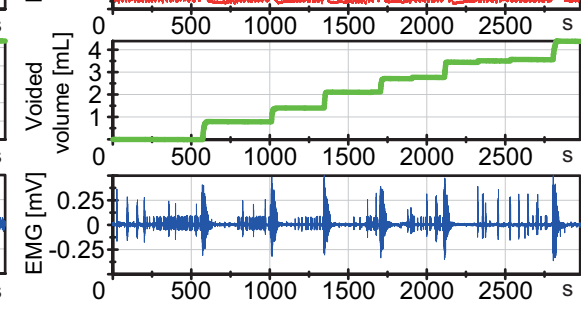

\section{f}
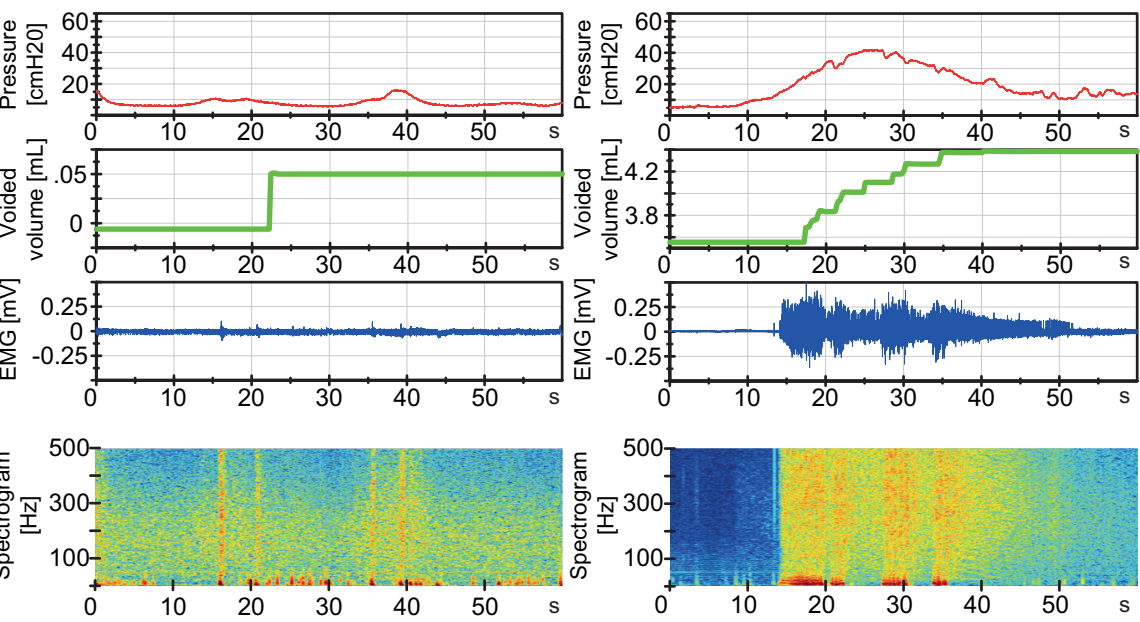

Figure 2. Bladder pressure, voided urine, and EUS-EMGs before (intact) and 7 and $28 \mathrm{~d}$ after incomplete SCI. DSD is fully developed at 4 weeks after injury. $\boldsymbol{a}, \boldsymbol{d}, 40 \mathrm{~min}$ ( $2200 \mathrm{~s}$ ) and $1 \mathrm{~min}$ ( $60 \mathrm{~s}$ ) windows of a representative urodynamic tracing from an intact rat with bladder catheter and EUS-EMG showing filling/voiding cycles. Top, Bladder pressure. Middle, Urine secreted (weight). Bottom, External urethral sphincter EMG. Panels at base of figure, Heat plot of EMG frequency spectrogram where red represents a high power of the corresponding frequency at the current time point and blue represents low power. $\boldsymbol{b}, \boldsymbol{e}$, Spinal cord-injured rat at 1 week after injury shows a flaccid bladder with continuous flow of urine and absent sphincter activity. $\boldsymbol{c}, \boldsymbol{f}$, Typical characteristics of DSD at 4 weeks after injury. During the filling phase, there are nonvoiding contractions with increasing magnitude as well as an increase in EUS-EMG activity during voiding.

dissected and postfixed for $24 \mathrm{~h}$ at $4^{\circ} \mathrm{C}$ in a solution containing $4 \%$ paraformaldehyde and $15 \%$ saturated picric acid for immunohistochemistry, or $4 \%$ paraformaldehyde (Sigma-Aldrich) in $0.1 \mathrm{~m}$ phosphate buffer, $\mathrm{pH}$ 7.4, for RNAScope in situ hybridization. Afterward, spinal cords were transferred to $30 \%$ sucrose in $0.1 \mathrm{M}$ phosphate buffer, $\mathrm{pH} 7.2$, and stored for $3 \mathrm{~d}$ for cryoprotection. The tissue was embedded in Tissue-Tek OCT compound, frozen in 2-methylbutane (Sigma-Aldrich) cooled to $-40^{\circ} \mathrm{C}$ with liquid nitrogen, and stored at $-20^{\circ} \mathrm{C}$ until further processing. For immunohistological analysis, $40-\mu$ m-thick L6-S1 spinal cord cross sections were cut on a cryostat and collected on slides (Huberlab, Superfrost Plus), and kept at $-20^{\circ} \mathrm{C}$ until further processing. For in situ hybridization analysis, $10-\mu$ m-thick L6-S1 spinal cord cross sections were collected free-floating in $4^{\circ} \mathrm{C}$ cold $0.1 \mathrm{~m}$ phosphate buffer and kept in antifreeze solution ( $15 \%$ sucrose, $30 \%$ ethylene glycol in $50 \mathrm{~mm}$ phosphate buffer) at $-20^{\circ} \mathrm{C}$ until further processing. For histological lesion site analysis, T7-T10 spinal cord sections were mounted on slides, dried on a heating plate at $60^{\circ} \mathrm{C}$ for $10 \mathrm{~min}$, and then stored at $-20^{\circ} \mathrm{C}$ until further processing.

Assessment of lesion completeness. Coronal $40 \mu \mathrm{m}$ cryostat serial sections of the injury site were manually reconstructed based on Nissl staining (cresyl violet) in Photoshop software (Adobe). The percentage of spared white matter at the largest lesion extent was calculated. Initial comparison of Nissl staining and neurofilament-160 immunohistochemical staining showed a very high correlation between the two protocols (see Fig. $7 a-c$ ). Low values of spared white matter were arduous to categorize into complete or incomplete groups based on the images. This, together with the fact that animals with $<3 \%$ spared white matter were equally distributed between the groups, led to the conclusion that rats with $<3 \%$ spared white matter had a complete lesion.
Immunohistochemistry. On-slide spinal cord cross sections were thawed for $10 \mathrm{~min}$ and then washed three times with $0.1 \mathrm{M}$ phosphate buffer, $\mathrm{pH}$ 7.2. Afterward, they were permeabilized in TNB [0.5\% TopBlock (LubioScience) in $0.1 \mathrm{M}$ Tris] containing $0.2 \%$ Triton X-100 for 60 $\mathrm{min}$ at room temperature, and subsequently incubated with the primary antibody (rabbit-anti-CRF, 1:400; Salk Institute) diluted in TNB and hypotonic $0.01 \mathrm{M}$ phosphate buffer containing $0.05 \%$ Triton X-100 at $4^{\circ} \mathrm{C}$ for $24 \mathrm{~h}$. The sections were then washed three times in $0.1 \mathrm{~m}$ PBS for $10 \mathrm{~min}$ each and incubated with the secondary antibody (goat-antirabbit-Cy3, 1:300; Jackson ImmunoResearch Laboratories). Stained sections were washed three times in $0.1 \mathrm{M}$ phosphate buffer for $10 \mathrm{~min}$ each, finally incubated in $0.05 \mathrm{M}$ Tris, $\mathrm{pH} 8.0$, for $10 \mathrm{~min}$, air-dried overnight at $4^{\circ} \mathrm{C}$, and coverslipped with fluorescence mounting medium (Merck, Mowiol). Slides were stored at $4^{\circ} \mathrm{C}$ upon imaging.

The specificity of the signal was assessed by omitting the incubation step of the sections in the primary antibody.

RNAScope in situ hybridization. Sequences of target, preamplifier, amplifier, and label probes are proprietary and commercially available (Advanced Cell Diagnosis). Here, we used probes against rat glycine transporter $2\left(\mathrm{GlyT}_{2}\right.$, Slc6a5), glutamic acid decarboxylase $2\left(\mathrm{GAD}_{2}\right)$, and vesicular glutamate transporter 2 ( vGluT $_{2}$, Slc17a6).

Experimental protocols were conducted according to guidelines from Advanced Cell Diagnosis. Briefly, slides were thawed at room temperature for $15 \mathrm{~min}$. Subsequently, slides were treated with hydrogen peroxide (Advanced Cell Diagnosis) for $10 \mathrm{~min}$ and washed twice in water for 2 min each, before incubation in target retrieval buffer (Advanced Cell Diagnosis) for $10 \mathrm{~min}$ at $98-100^{\circ} \mathrm{C}$. Slides were washed first in water and then in $100 \% \mathrm{EtOH}$ (Reuss Chemie). Then protease treatment was applied for $30 \mathrm{~min}$ at $40^{\circ} \mathrm{C}$ in a HybEZ oven (Advanced Cell Diagnosis). 


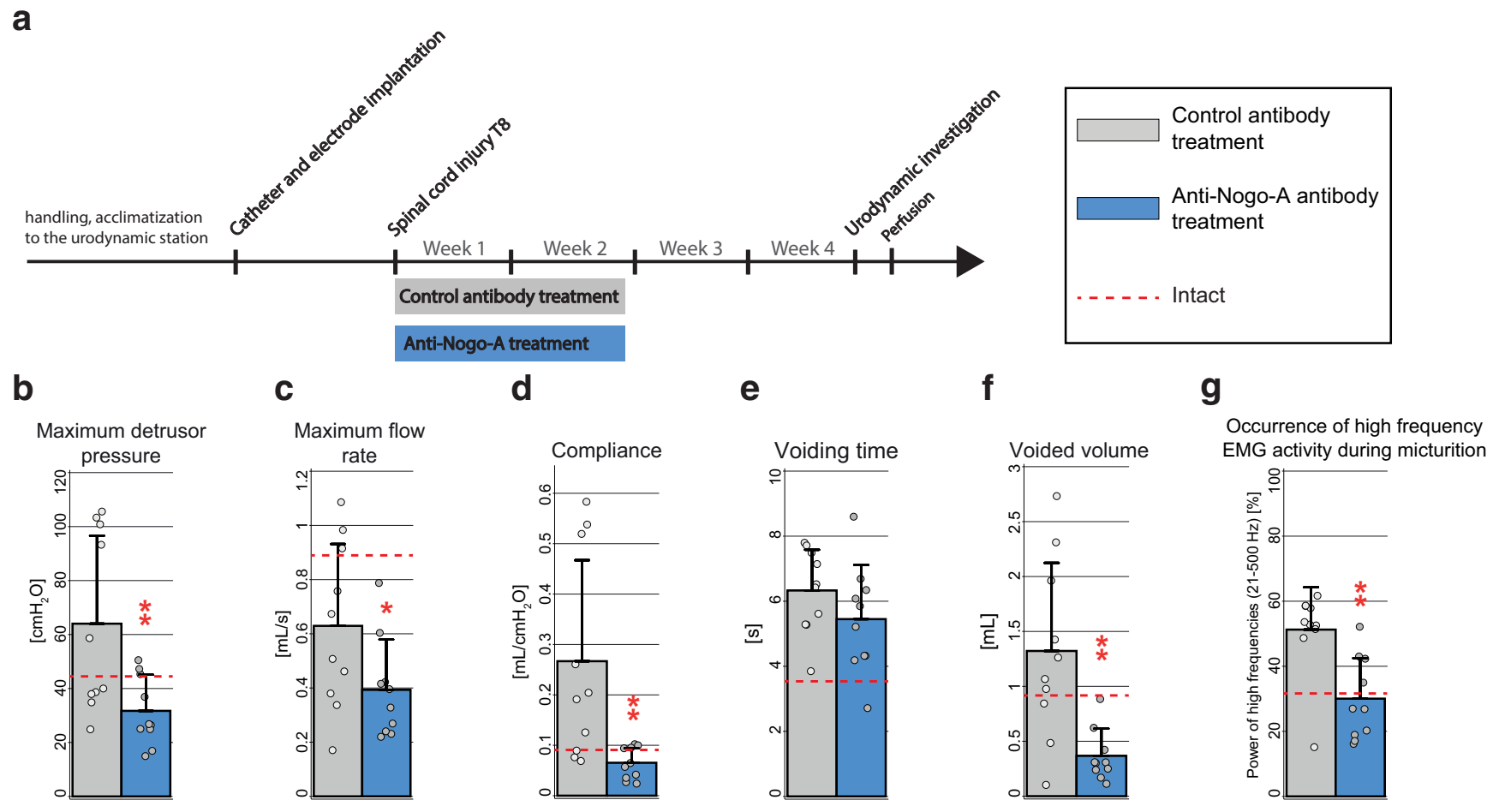

Figure 3. Anti-Nogo-A antibody effects on lower urinary tract function in incomplete $S \mathrm{Cl}$ assessed by urodynamics. $\boldsymbol{a}$, Study design. $\boldsymbol{b}$, Maximum detrusor pressure $\left[\mathrm{cmH} \mathrm{H}_{2} \mathrm{O}\right] . \boldsymbol{c}, \mathrm{Maximum}$ flow rate $[\mathrm{ml} / \mathrm{s}] . \boldsymbol{d}$, Bladder compliance [ml/ $\mathrm{cmH}_{2}$ 0].e, Voiding time [s].f, Voided volume [ml]. $\boldsymbol{g}$, EMG activity during voiding (Fig. $\left.1 j, k, s\right)$ compared with the whole period analyzed (Fig. $\left.1 j, k, \mathrm{u}\right)[\%]$. Statistical testing: data of anti-Nogo-A antibody-treated rats and control antibody-treated rats were analyzed with an unpaired Student's t test; ${ }^{*} p<0.05 ;{ }^{* *} p<0.01$, ${ }^{* * *} p<0.001$. Red dashed line reflects the values of intact animals.

Subsequently, sections were incubated in a mix containing the three hybridization probes for $2 \mathrm{~h}$ at $40^{\circ} \mathrm{C}$. After washing the slides twice in washing buffer (Advanced Cell Diagnosis), the three probes were amplified in a consecutive manner, with two washing steps in washing buffer (Advanced Cell Diagnosis) in between each amplification step. Further amplification steps were performed with HRP detecting the specific channel of the different probes, always with washing steps with washing buffer (Advanced Cell Diagnosis) in between. The signals were developed with TSA Plus fluorescein, TSA Plus Cyanin3, and TSA Plus Cyanin5 (PerkinElmer, TSA Cy3, Cy5, TMR, Fluorescein Evaluation kit) for the probe targeting $\mathrm{GlyT}_{2}, \mathrm{GAD}_{2}$, and $\mathrm{vGluT}_{2}$, respectively. After the development of the last probe, sections were washed twice in washing buffer (Advanced Cell Diagnosis) and counterstained with DAPI (Advanced Cell Diagnosis) for $45 \mathrm{~s}$ at room temperature. Slides were coverslipped using fluorescence mounting medium (Merck, Mowiol) and let dry overnight in the dark. Afterward, they were stored at $4^{\circ} \mathrm{C}$ upon imaging.

Quantification of immunofluorescent signal. The CRF signal was quantified by imaging three randomly chosen sections with a confocal microscope (40×; Olympus America, Olympus FV1000). Microscope and laser parameters, such as laser intensity, were optimized during the first imaging and kept constant across all sections. Mosaic pictures were imaged and processed with Olympus FV1000 software. Maximum intensity projections were created, and pictures were exported in TIFF format for investigation. Further analyses were performed with the Fiji image processing software (National Institutes of Health, ImageJ, version 1.0). Mean gray values for the regions of the dorsal horn, intermediolateral nucleus (IML), and dorsal gray commissure were measured in the three sections and background intensities obtained from the central canal, where no signal is expected, were subtracted for each region. The values were normalized to the equivalent values of the intact animals.

Quantification of RNAScope in situ hybridization. Five to seven sections per animal were imaged with a fluorescent microscope (20×; Zeiss, Axio Scan.Z1). Three randomly chosen pictures were analyzed with Fiji and a custom-made Matlab (The MathWorks, 2017b) script. Briefly, the coordinates of each stained cell were retrieved within Fiji, and then used to plot the cells to a standardized spinal cord cross-section template with Matlab, thereby normalizing the analysis to potential tissue distortion. The number of $\mathrm{Gly}_{2}$-positive, $\mathrm{GAD}_{2}$-positive, and vGluT $\mathrm{T}_{2}$-positive cells was calculated within the laminae 1,2 , and 3 , which reflect the dorsal horn; laminae 4 and 5, which comprehend the IML; and lamina X (Lam $\mathrm{X})$, comprising the dorsal gray commissure.

Statistical analysis. Data are reported as mean \pm SD. Quantile-quantile plots were generated of all data and visually assessed. All data are assumed to be approximately normally distributed. For the comparison of the impact of SCI on bladder function by urodynamics, immunohistochemistry, and in situ hybridization, data were analyzed with a one-way repeated-measures ANOVA followed by Bonferroni's post hoc testing. For comparison of unrelated samples, an unpaired $t$ test was used. The value of significance was considered at $p<0.05$. A priori power calculations were performed with $\alpha$ being 0.05 and a power of $80 \%$. Statistical analyses were performed using Stata statistical software, version 14 (StataCorp).

\section{Results}

\section{Lower urinary tract dysfunction following SCI in adult rats}

Animals were injured at day 0 and lower urinary tract function was assessed once a week for 4 weeks (Fig. 1a) during urodynamic measurements. On day 3-5 after the lesion, all rats in the SCI groups (complete and incomplete lesions) showed a complete initial paralysis of both hindlimbs as assessed by the BBB score (Basso, Beattie, and Bresnahan; data not shown; Basso et al., 1995). The T8-level bilateral partial transections interrupted between 2 and 55\% of the spinal cord white matter (see Fig. 7); spared white matter was mostly found in the ventral and ventrolateral funiculi, often unilateral only. One week after injury, most rats of the incomplete lesion groups started to develop hindlimb movements resulting in a BBB score of $>103$ weeks after injury. Completely spinal cord-injured animals showed a persisting se- 
a

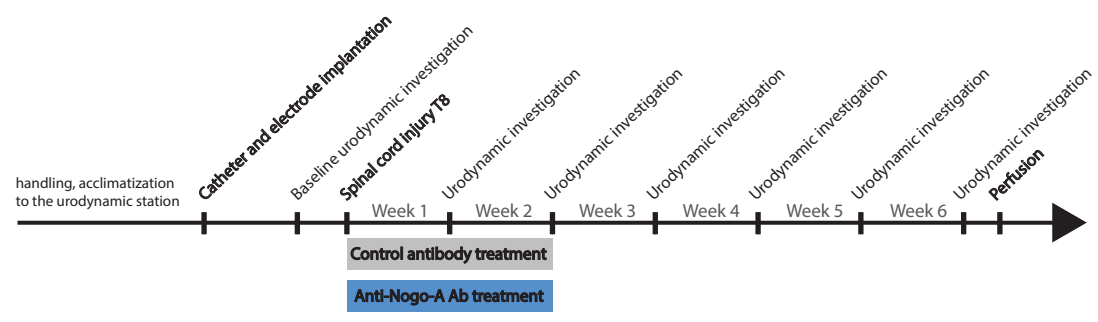

C

d

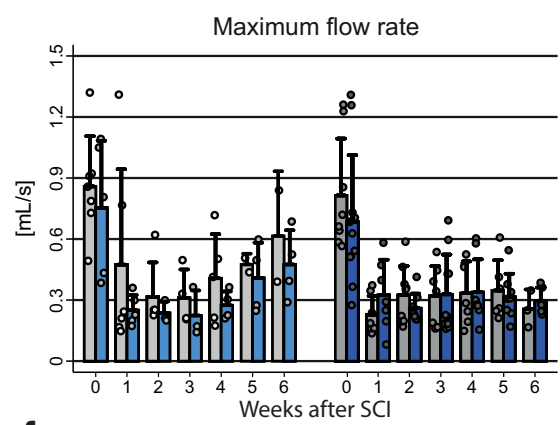

f

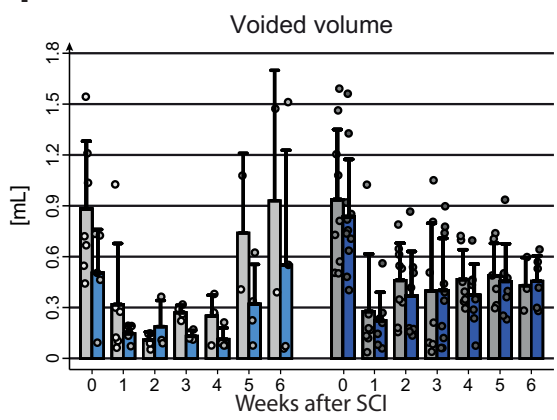

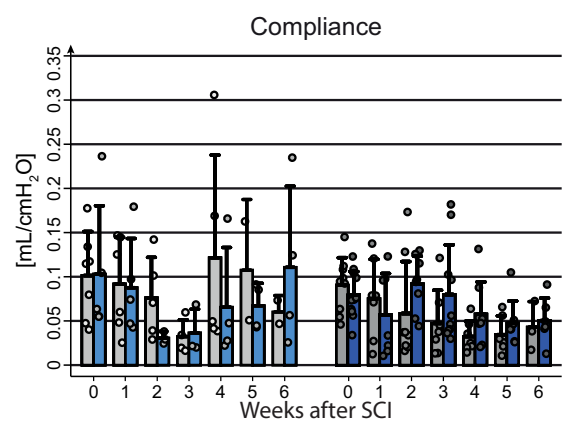
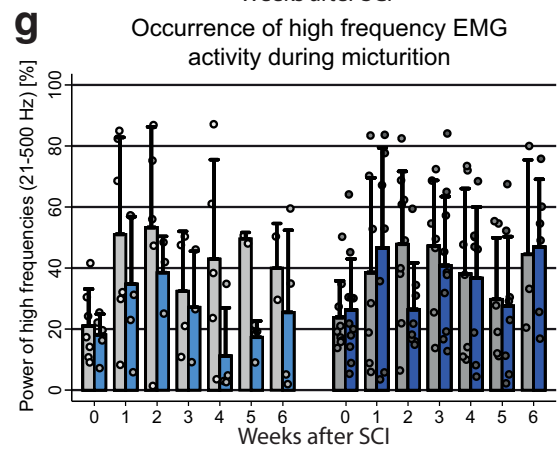

b

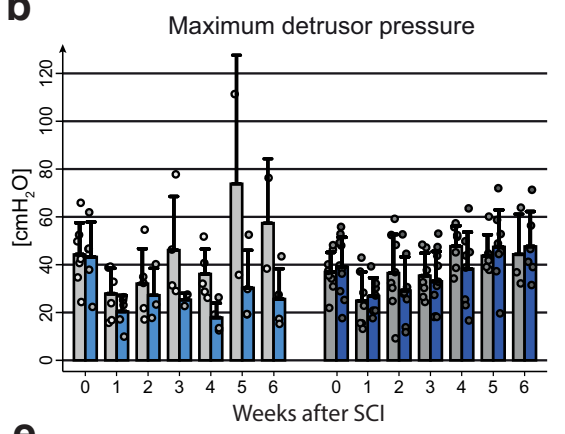

e

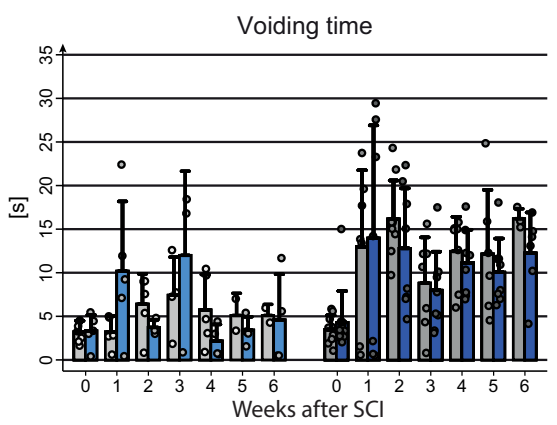

Control Ab treatment incomplete spinal cord injury

Anti-Nogo-A Ab treatment, incomplete spinal cord injury

Control Ab treatment, complete spinal cord injury

Control Ab treatment complete spinal cord injury

Figure 4. Anti-Nogo-A antibody effects on lower urinary tract function in incomplete versus complete SCl assessed by urodynamics. $\boldsymbol{a}$, Study design. $\boldsymbol{b}, \mathrm{Maximum}$ detrusor pressure $\left[\mathrm{cmH}_{2} 0\right]$. $\boldsymbol{c}$, Maximum flow rate $[\mathrm{ml} / \mathrm{s}]$.d, Bladder compliance [ml/cmH $\mathrm{cm}_{2}$ ] . e, Voiding time [s].f, Voided volume [ml]. g, EMG activity during voiding (Fig. 1j,k, s) compared with the whole period analyzed (Fig. $1 j, k, u)[\%] ;$ no statistical testing was performed on this study.

vere impairment of hindlimb function until the end of the experiment, i.e., $\leq 4$ weeks after injury. The impairments observed in the rats are comparable to human SCI patients with American Spinal Injury Association impairment scale (AIS) A for the complete SCI group and AIS C for the incomplete SCI group (www.ais.emsci.org).

In all groups, bladder function was absent during the first week after injury and, very much in contrast to locomotor performance, showed only very limited spontaneous recovery. In both SCI groups, maximum detrusor pressure at $7 \mathrm{~d}$ after injury dropped significantly $(p<0.05)$ to approximately half of its value before SCI (Fig. 1b). Over the following 3 weeks, maximum detrusor pressure constantly increased, exceeding the baseline measurements at 4 weeks after injury. However, maximum detrusor pressure also increased in the intact group, reaching a value of $164 \%$ of the baseline, a phenomenon that may be related to the chronic implantation of a bladder catheter. During voiding, maximum flow rates dropped to half of the preinjury values in both SCI groups (Fig. 1c). Bladder compliance was reduced in both SCI groups at 4 weeks after injury (Fig. 1d). Voiding time increased from 2 weeks after injury on (Fig. 1e). Voided volume was strongly and significantly reduced after injury, showing a certain degree of recovery but remaining significantly below baseline values in both injury groups until the end of the experiment (Fig. 1f). The intact group showed an increase of the voided volume over time compared with prelesion baseline. All these urodynamic findings are highly consistent with urodynamic observations made in human SCI patients and mirror the human acute and early chronic stages after SCI (Groen et al., 2016).

\section{SCI induced changes in EUS-EMG activity}

In intact rats, the EMG of the EUS showed strong peak activity in the high-frequency range $(21-500 \mathrm{~Hz}$, representing mainly striated muscle activity; Solomonow et al., 1990) at the high filling state immediately preceding voiding, as well as after voiding. High-frequency activity was low during voiding (Figs. $1 j, 2 a, d$ ), whereas in contrast, slow-wave bursting activity $(2-20 \mathrm{~Hz}$, representing mainly smooth muscle activity; Domino et al., 2017) was prominent (Figs. 1j, 2a,d).

One week after injury, all spinal cord-injured rats suffered from urinary retention and were unable to void by themselves. Urodynamic assessments confirmed this observation showing an acontractile bladder in all rats, which led to overflow in- 


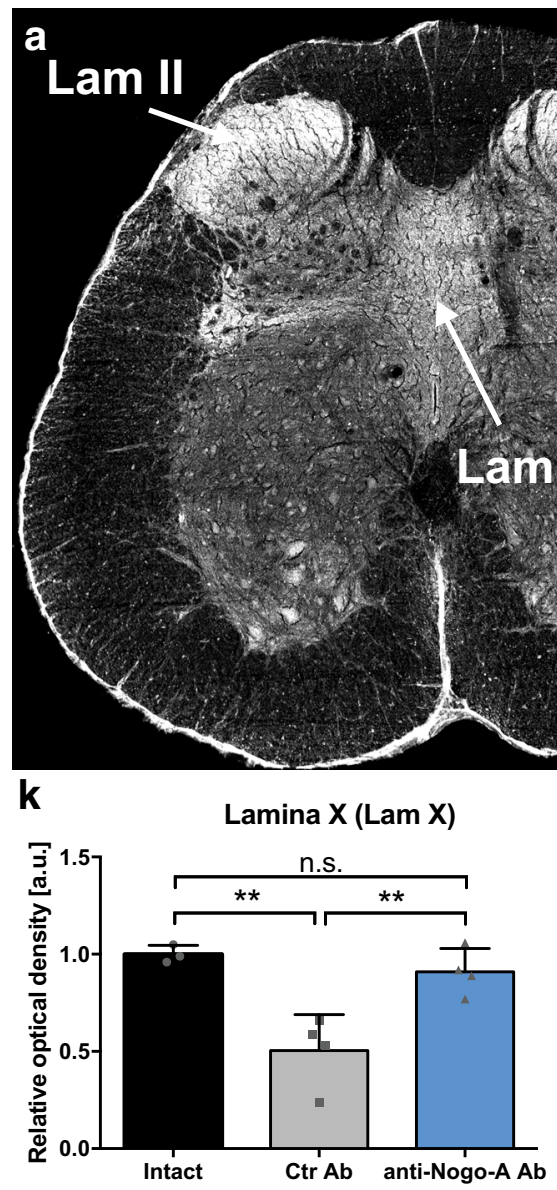

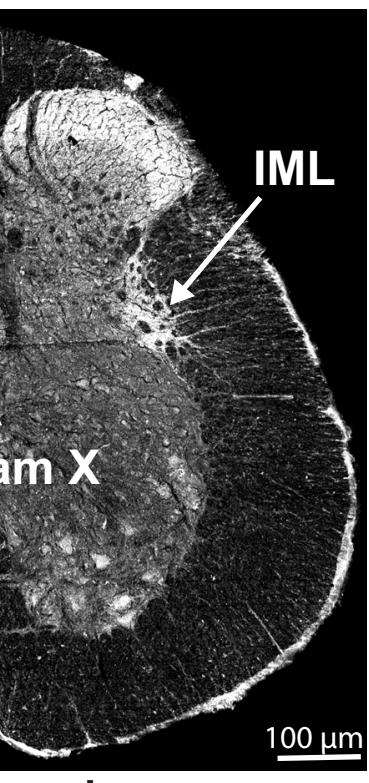
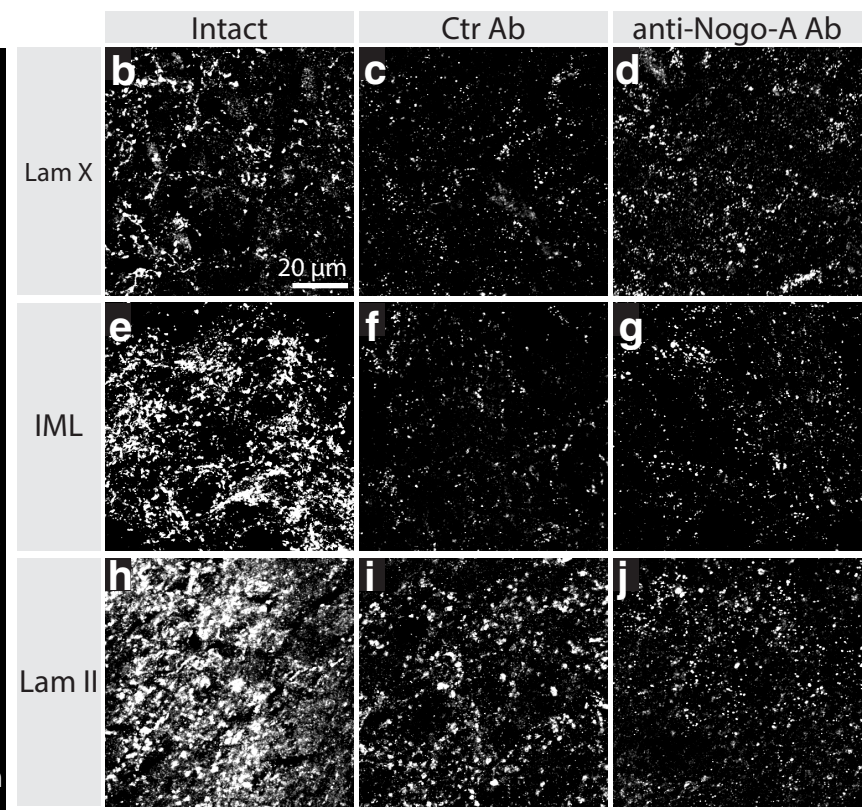

$\mathrm{m}$
Intermediolateral column (IML)

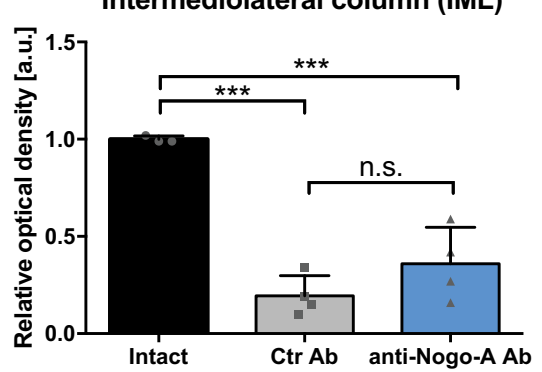

Figure 5. Innervation of lumbosacral spinal cord by CRF-positive fibers, including bulbospinal fibers from the PMC at $28 \mathrm{~d}$ after injury. $\boldsymbol{a}$, In intact rats, immunofluorescent CRF-positive fibers and terminals are concentrated in dorsal horn Lam II, the IML, and Lam X. $\boldsymbol{b}$-j, High-magnification image (40X) of Lamina X ( $\boldsymbol{b}-\boldsymbol{d})$, IML column ( $\boldsymbol{e}-\boldsymbol{g})$, and Lam II ( $\boldsymbol{h}-\boldsymbol{j})$ in intact, spinal cord-injured control antibody-treated (Ctr Ab), and injured anti-Nogo-A antibody-treated rats. $\boldsymbol{k}-\boldsymbol{m}$, Relative optical density quantitation for CRF in Lam X, IML, and Lam II. Data are presented as means \pm SD. Scale bars: $\boldsymbol{a}, 100 \mu \mathrm{m} ; \boldsymbol{b}-\boldsymbol{j}, 20 \mu \mathrm{m} ; \mathrm{ns}$, not significant; ${ }^{*} p<0.05 ;{ }^{* *} p<0.01 ;{ }^{* * *} p<0.001$.

continence once the bladder reached its maximum capacity (Fig. 2b,e).

Sixteen of 17 rats with either complete or incomplete SCI developed DSD, starting 1-2 weeks after the lesion, characterized by tonic high-frequency sphincter EMG activity $(21-500 \mathrm{~Hz})$ during the voiding phase. This resulted in interrupted urine release and highly prolonged voiding phases (Figs. $1 k, 2 c, f$ ). These characteristic features again closely resemble the situation seen in severely spinal cord-injured human patients.

Urodynamic and EUS parameters were not influenced by lesion completeness

No significant differences were found in any urodynamic parameter when the large, incomplete lesions and complete spinal cordinjured animals were compared (Fig. $1 b-f$ ). Also, we did not find differences in high-frequency activity of the EUS EMG during voiding (Fig. $1 h-i$ ) between incomplete and complete SCI groups.

Effects of anti-Nogo-A antibody treatment on neurogenic lower urinary tract dysfunction after incomplete SCI

Anti-Nogo-A antibody therapy significantly reduced important urodynamic abnormalities in incompletely spinal cord-injured animals when compared with incompletely lesioned animals treated with control antibody (Fig. 3a). Four weeks after SCI, we observed significant changes toward more physiological values in maximum detrusor pressure (Fig. $3 b ; p=0.01$ ), maximum flow rate (Fig. $3 c ; p=0.05$ ), compliance (Fig. $3 d ; p=0.006$ ), and voided volume (Fig. $3 f ; p=0.002$ ) of anti-Nogo-A-treated SCI animals. Voiding time 4 weeks after injury did not differ between the treated and control group (Fig. $3 e ; p>0.05$ ). Anti-Nogo-A antibodies also significantly reduced EUS activity during voiding in incompletely spinal cord-injured animals compared with incompletely lesioned animals treated with control antibody. Four weeks after SCI, we found a significant reduction in the proportion of high-frequency EMG values during voiding $(p=0.002)$ in the anti-Nogo-A antibody-treated animals when compared with control antibody-treated spinal cord-injured animals (Fig. $3 g$ ).

Anti-Nogo-A-treatment effects on neurogenic lower urinary tract dysfunction after complete versus incomplete SCI We further investigated whether the observed effect of antiNogo-A antibody therapy in incompletely spinal cord-injured rats could be replicated in animals with a complete SCI (Fig. 4a). Anti-Nogo-A antibody-treated animals showed again a trend toward a more pronounced treatment effect in incompletely spinal cord-injured animals compared with incompletely lesioned animals treated with control. Several of the urodynamic abnormalities were reduced starting 2-3 weeks after SCI (Fig. $4 b-g$ ). Four 

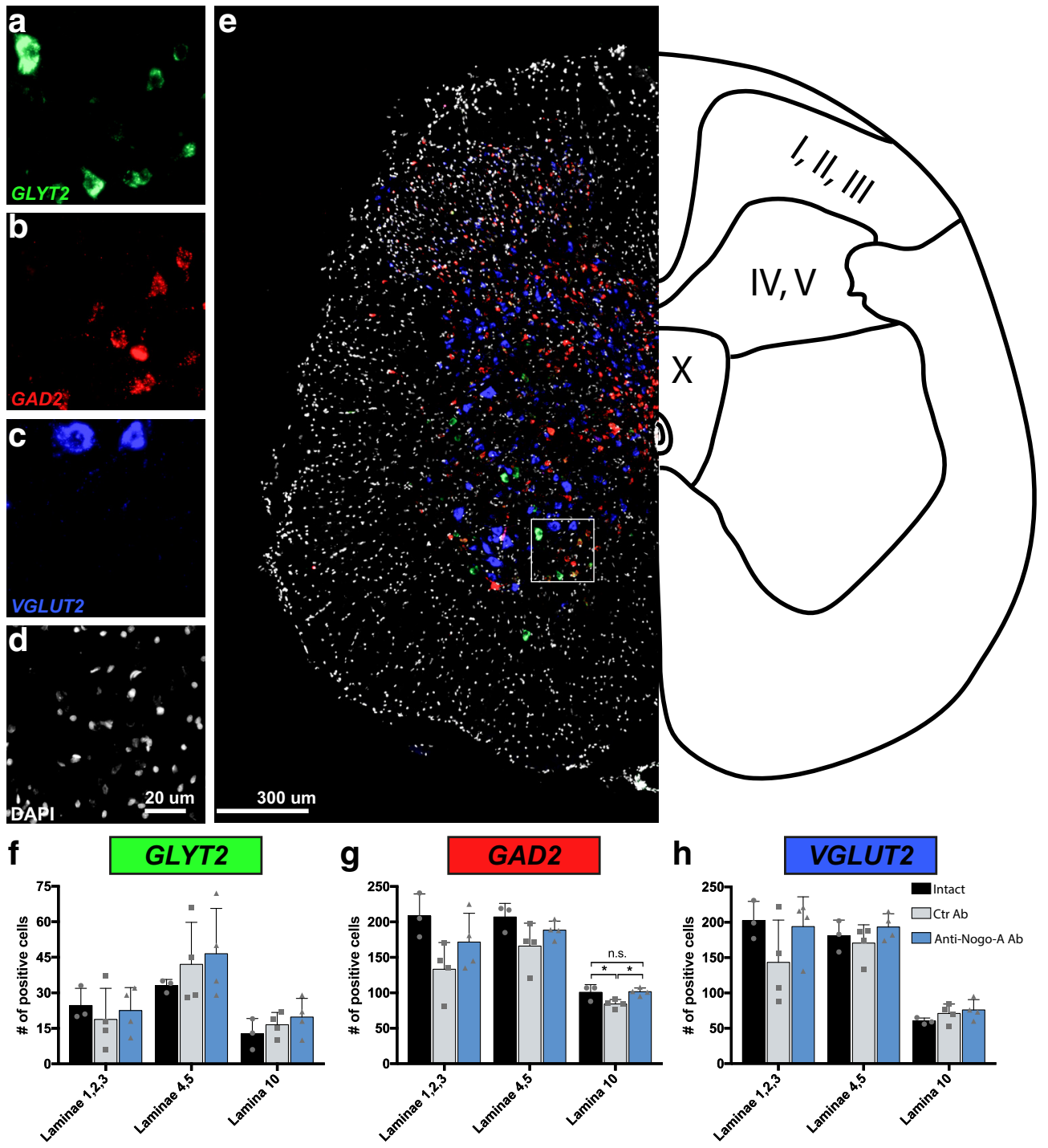

Figure 6. Glutamatergic, GABAergic, and glycinergic neurons in the sacral cord of intact and spinal cord injured, antibody-treated rats at $28 \mathrm{~d}$ after injury. $\boldsymbol{a}-\boldsymbol{e}$, Representative images of fluorescent in situ hybridization for mRNA of $\operatorname{GlyT}_{2}(\boldsymbol{a}), \mathrm{GAD}_{2}(\boldsymbol{b})$, and $\mathrm{VGlut}_{2}(\boldsymbol{c})$. $\boldsymbol{d}$, Sections were counterstained with DAPI. $\boldsymbol{e}$, Representative image of the three merged probes, indicating the laminae in the three analyzed regions (I-III; IV, V; X). $\boldsymbol{f}$ - $\boldsymbol{h}$, Quantification of glycinergic $(\boldsymbol{f})$, GABAergic $(\boldsymbol{g})$, and glutamatergic $(\boldsymbol{h})$ neurons in the sacral cord of intact animals, incomplete spinal cord-injured animals treated with control antibody ( $(\mathrm{tr} A b)$, and incomplete spinal cord-injured animals treated with anti-Nogo-A antibodies (anti-Nogo-A). Data are presented as means \pm SD. Scale bars: $\boldsymbol{a}-\boldsymbol{d}, 20 \mu \mathrm{m} ; \boldsymbol{e}, 300 \mu \mathrm{m} ; \mathrm{ns}$, not significant; ${ }^{*} p<0.05$.

weeks after SCI, the anti-Nogo-A antibody-treated group showed a reduction in maximum detrusor pressure (50\%; Fig. 4b), maximum flow rate (32\%; Fig. $4 c)$, compliance (46\%; Fig. $4 d$ ), voiding time (62\%; Fig. 4e), and voided volume (34\%; Fig. $4 f$ ) when compared with the control antibody-treated group. The proportion of high-frequency EMGs during voiding showed the biggest change; it was decreased by $73 \%$ in the anti-Nogo-A antibodytreated animals (Fig. 4g). Very much in contrast to these group differences in the incompletely lesioned rats, no effect of antiNogo-A therapy on lower urinary tract function was observed in animals with a complete SCI (Fig. $4 b-g$ ). However, no statistical testing was performed in this experiment due to the restricted number of animals per group.

Neuroanatomical changes in the lumbosacral spinal cord after injury and anti-Nogo-A antibody treatment

The neuropeptide CRF is highly enriched in PMC neurons projecting to the lumbosacral spinal cord (Valentino et al., 1994; Verstegen et al., 2017). Immunofluorescent stainings for CRF in cross sections of the L6-S1 spinal cord revealed three main target regions innervated by CRF-positive fibers: lamina II of the dorsal horn (Lam II), the IML, and Lam X of the ventral gray matter (Fig. 5a). Interestingly, the IML contains the preganglionic autonomic motoneurons responsible for bladder contraction (De Groat and Ryall, 1968; Nadelhaft and Booth, 1984), while the Lam X contains interneurons that were shown to modulate the activity of somatic motoneurons innervating the bladder and EUS (Marson, 1997; Nadelhaft and Vera, 2001). The observed CRF signal in Lam II is believed to originate from innervating peripheral sensory fibers (Skofitsch et al., 1985; Kim et al., 2011), since previous anterograde tracings from the PMC did not result in labeling of this region (Verstegen et al., 2017). Four weeks after incomplete SCI, animals treated with control antibodies showed a strong, significant decrease in CRF-positive innervation of Lam $\mathrm{X}$ compared with noninjured animals $(p=0.004)$. In contrast, the density of CRF-positive fibers and terminals in this region in the injured anti-Nogo-A antibody-treated rats was not different from that of intact rats $(p=0.99)$, but was significantly higher 
a

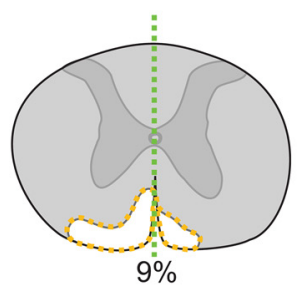

d

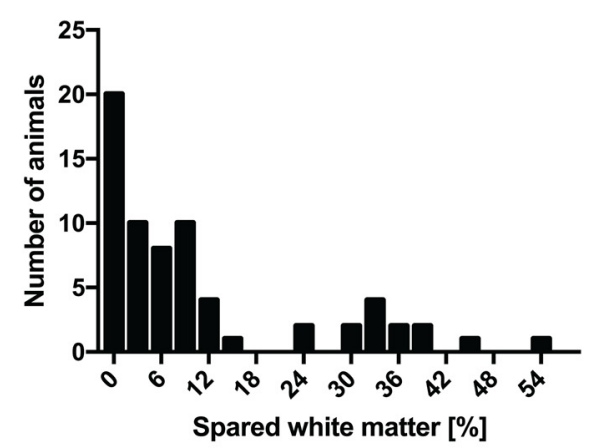

b

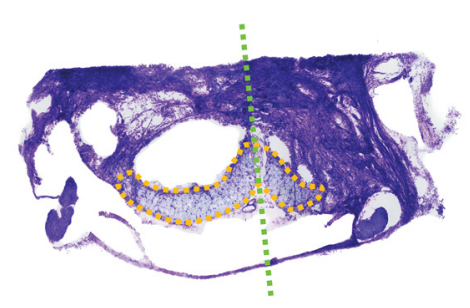

C

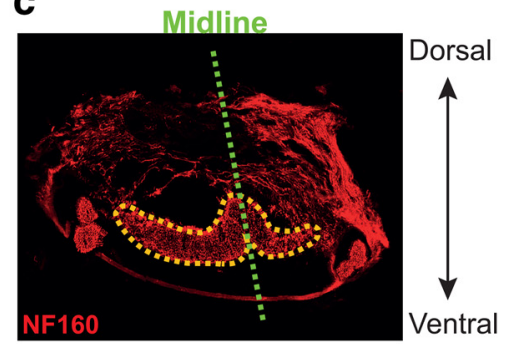

e

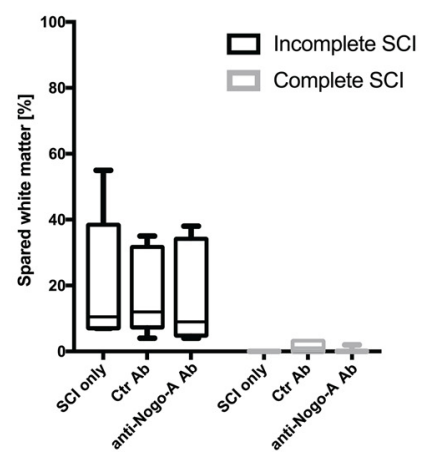

Figure 7. Spinal cord lesion assessment and distribution among groups. $\boldsymbol{a}$, Schematic reconstruction of a spinal cord lesion. $\boldsymbol{b}$, Representative Nissl staining of a spinal cord used to reconstruct the lesion size. c, Neurofilament-160 immunohistological staining shows a high degree of correlation with the Niss staining. $\boldsymbol{d}$, Distribution of lesion sizes across the different animals. $\boldsymbol{e}$, Comparison of the spared white matter between animals with a SCI group and no treatment (SCl only), spinal cord-injured rats treated with control antibody ( $(\mathrm{Ctr}$ Ab), and spinal cord-injured animals treated with anti-Nogo-A antibody (anti-Nogo-A) in case of a complete (black) or incomplete (gray) lesion. Data in e are shown as median and 10-90 percentile.

than that of the control antibody spinal cord-injured animals (Fig. $5 b-d, k ; p=0.009$ ). In the IML region, both injury groups showed a reduced CRF-positive fiber density compared with intact animals (Fig. 5e-g, $m ; p=0.0001$ and 0.0006 , respectively). Anti-Nogo-A antibody-treated rats showed a trend for higher values (Fig. 5). In contrast, no difference was observed between the two injury groups $(p=0.33)$ in the Lam II CRF innervation, which significantly decreased in both spinal cord-injured groups when compared with intact rats (Fig. $5 h-j, l ; p=0.02$ ).

We further analyzed the numbers of glycinergic, GABAergic, and glutamatergic interneurons located in the segments L6-S1 of the spinal cord using in situ hybridization for the respective cell type-specific markers GLYT2, GAD2, and VGLUT2 mRNAs. No differences in the number of glycinergic cells expressing GLYT2 mRNA were observed between intact animals and rats with large but incomplete SCIs treated with either control or anti-Nogo-A antibodies in any of the regions analyzed, i.e., Lam I-III, IV-V, and $\mathrm{X}$ (Fig. $6 a, e, f$ ). The number of GABAergic, GAD2 mRNApositive cells showed a trend toward a decrease in cell number in all areas analyzed. However, only the dorsal gray commissure area showed a small but significant reduction in GAD2-positive neurons in the spinal cord-injured, control antibody-treated rats when compared with intact animals $(p=0.04)$. Interestingly, this reduction in cell number was not present in the anti-Nogo-A antibody-treated SCI group (Fig. 6b,g). Furthermore, we did not observe any difference between groups in the number of glutamatergic, VGLUT2-positive neurons in Lam I-III, IV-V, nor X (Fig. $6 c, e, i)$.

\section{Discussion}

A massive increase in EMG activity of the EUS during the voiding phase was the most prominent change observed using urodynamic measurements in adult rats with either an incomplete or complete lesion in the low thoracic spinal cord. This wrongly timed increase in EUS-EMG activity is a hallmark of DSD, which is defined by a dyssynergistic contraction of the EUS during voiding, resulting in increased bladder pressure and therefore compromised voiding. In humans, this often results in urinary reflux to the kidneys and over time to life-threatening kidney damage (Panicker et al., 2015). Interestingly, as in patients with SCI, the characteristic DSD features in rats developed over time, i.e., at 2-4 weeks after injury, which is very much in line with the human condition (1-6 months in humans; Bywater et al., 2018). Our findings in rats are therefore highly translational and comparable to data from human SCI patients (Weld et al., 2000; Fowler et al., 2008; Schöps et al., 2015). Intrathecal administration of antiNogo-A antibodies for 2 weeks after a large but incomplete T8 SCI resulted in a reduction of the impairment of several key urodynamic functions at 4 weeks after lesion. For example, the EMG overactivity of the EUS during voiding recovered nearly back to prelesion baseline levels. This was in great contrast to control antibody-treated SCI animals. However, no treatment effect was observed in animals with a complete SCI treated with antiNogo-A antibody (Fig. 7). These findings indicate a crucial role of the remaining descending ventral tract fibers $(4-38 \%$ of white matter sparing in our study) in the anti-Nogo-A antibody treatment-enhanced restoration of bladder function.

In human spinal cord-injured patients, the development of neurogenic lower urinary tract dysfunction after SCI follows a typical time course, which is characterized by an initial phase of detrusor and sphincter muscle inactivity (flaccid bladder), resulting in incontinence and high postvoid residuals (Panicker et al., 2015). A very similar situation was encountered in our rats in all the experimental SCI groups at $7 \mathrm{~d}$ after injury. Absence of descending control and spinal shock are the most likely reasons for this condition. Detrusor overactivity and simultaneous abnormal contractions of the EUS during voiding (Abrams et al., 2002) 
then lead to increased intravesical pressure. In our animal model, the incomplete thoracic SCI resulted in the development of characteristic DSD features within the first 4 weeks following injury. Impairment of most of the urodynamic and EUS-EMG parameters was much more pronounced after an incomplete lesion than after a complete SCI. This finding points to an important role of spared descending fibers and their potential detrimental, spontaneous plastic changes after partial injury for the initial development of DSD.

Intrathecal delivery of function-blocking anti-Nogo-A antibodies, which have been shown to promote nerve-fiber sprouting and regeneration in the adult mammalian CNS (Liebscher et al., 2005; Lindau et al., 2014), for the first 2 weeks after the lesion had a strong positive effect on the recovery of bladder function. Several of the key urodynamic and electrophysiological parameters showed significant and pronounced improvement in the antiNogo-A antibody-treated compared with the control antibodytreated SCI group. Most strikingly, the anti-Nogo-A antibody treatment led to a pronounced recovery of the physiological EUS function during voiding. The beneficial effects of anti-Nogo-A antibodies on the lower urinary tract are evident. Nevertheless, we could observe a few peculiarities. Animals treated with antiNogo-A antibodies had a smaller voided volume, which was caused by an increased micturition frequency. Furthermore, the flow rate of these animals was reduced when compared to control treated animals, but this was expected since the intravesical pressure was smaller. Anti-Nogo-A antibody treatment has been shown to induce regeneration and sprouting of the corticospinal tract (Liebscher et al., 2005; Freund et al., 2006) and restoration of the serotonergic projection density (Müllner et al., 2008) after large but incomplete SCI in several species, including macaque monkeys. Immunohistochemical staining against the neuropeptide CRF enabled us to specifically visualize descending projections from the PMC to the lumbosacral spinal cord (Skofitsch et al., 1985; Kim et al., 2011). In line with existing literature, animals with an SCI showed a massive decrease in CRF-positive fibers in the lumbosacral spinal gray matter at 4 weeks after injury (Studeny and Vizzard, 2005). Interestingly, animals treated with the anti-Nogo-A antibodies during the first 2 weeks after injury showed CRF-fiber densities comparable to intact rats in Lam X, but not in Lam II or in the IML, where the PMC-fiber loss was comparable to that of the control antibody-treated SCI rats. This suggests that, in the anti-Nogo-A antibody-treated animals, the spared descending fibers from the PMC sprouted below the level of the injury in a specific target region, Lam X, thereby restoring functional, supraspinal input from the key bladder control system located in the brainstem. Keller and colleagues recently showed that in addition to the CRF-positive neurons, the PMC contains an additional population of neurons positive for the estrogen receptor $1 \alpha$. This estrogen receptor $1 \alpha$-positive neuronal population in the PMC is crucial for the correct function of the EUS and thereby for voluntary control of micturition (Keller et al., 2018). Thus, CRF-positive fibers might not be the only key players in the observed recovery of lower urinary tract function following anti-Nogo-A antibody therapy. In fact, a number of additional changes in the circuitry underlying the control of bladder function will be enhanced by the temporary neutralization of the neurite growth inhibitory factor Nogo-A and thereby might contribute to the reconfiguration of the bladder control on spinal and supraspinal levels (Bareyre et al., 2004; Filli et al., 2014). Our results are also in line with an earlier study that found that rats with slightly smaller incomplete lesions recovered functional bladder control earlier when treated with anti-Nogo-A antibodies (Liebscher et al., 2005).

Earlier studies investigating the neural circuits involved in lower urinary tract function showed that the PMC projects to GABAergic and glycinergic interneurons in the lumbosacral cord (Blok and Holstege, 2000; Sie et al., 2001), and that an SCI causes a downregulation of glycine and GABA in the spinal cord and lumbosacral dorsal root ganglia of rats (Miyazato et al., 2003, 2008a). Furthermore, spinal cord-injured animals affected by detrusor overactivity and DSD showed some degrees of recovery after dietary supplementation of glycine or after intrathecal application of $\mathrm{GABA}_{\mathrm{A}}$ or $\mathrm{GABA}_{\mathrm{B}}$ agonists (Miyazato et al., 2005, 2008b). These results indicate that inhibitory intraspinal connectivity is essential to coordinate bladder and sphincter function. Our results also suggest that these interneuron populations in the lumbosacral cord tend to decrease in number after injury, possibly due to a lack of descending inputs and therefore proper synaptic innervation. The decrease in numbers of GABAergic interneurons was dampened in the animals treated with antiNogo-A antibodies. This can most likely be explained by antiNogo-A antibody treatment enhancing the sprouting of spared descending fibers below the injury site, thereby restoring critical synaptic inputs to the interneurons.

A human phase-I safety and tolerability trial with intrathecal application of anti-Nogo-A antibodies in acutely spinal cordinjured patients has been concluded successfully (Kucher et al., 2018). A phase-two randomized controlled European multicenter trial testing for upper-limb motor recovery in acute tetraplegic patients is ongoing (https://nisci-2020.eu). Bladder parameters will be monitored as part of the panel of secondary readouts in this trial. Data addressing potential beneficial effects of Nogo-A suppression after SCI in humans should therefore become available soon.

\section{References}

Abrams P, Cardozo L, Fall M, Griffiths D, Rosier P, Ulmsten U, van Kerrebroeck P, Victor A, Wein A, Wein A (2002) The standardisation of terminology of lower urinary tract function: report from the standardisation sub-committee of the international continence society. Neurourol Urodyn 21:167-178.

Bacsu CD, Chan L, Tse V (2012) Diagnosing detrusor sphincter dyssynergia in the neurological patient. BJU Intl 109 [Suppl 3]:31-34.

Bareyre FM, Kerschensteiner M, Raineteau O, Mettenleiter TC, Weinmann O, Schwab ME (2004) The injured spinal cord spontaneously forms a new intraspinal circuit in adult rats. Nat Neurosci 7:269-277.

Basso DM, Beattie MS, Bresnahan JC (1995) A sensitive and reliable locomotor rating scale for open field testing in rats. J Neurotrauma 12:1-21.

Blok BF, Holstege G (2000) The pontine micturition center in rat receives direct lumbosacral input. an ultrastructural study. Neurosci Lett 282:2932.

Bywater M, Tornic J, Mehnert U, Kessler TM (2018) Detrusor acontractility after acute spinal cord injury-myth or reality? J Urol 199:1565-1570.

Cheng CL, de Groat WC (2004) The role of capsaicin-sensitive afferent fibers in the lower urinary tract dysfunction induced by chronic spinal cord injury in rats. Exp Neurol 187:445-454.

De Groat WC, Lalley PM (1972) Reflex firing in the lumbar sympathetic outflow to activation of vesical afferent fibres. J Physiol 226:289-309.

De Groat WC, Ryall RW (1968) The identification and characteristics of sacral parasympathetic preganglionic neurones. J Physiol 196:563-577.

de Groat WC, Kawatani M, Hisamitsu T, Cheng CL, Ma CP, Thor K, Steers W, Roppolo JR (1990) Mechanisms underlying the recovery of urinary bladder function following spinal cord injury. J Auton Nerv Syst 30:S71S77.

de Groat WC, Griffiths D, Yoshimura N (2015) Neural control of the lower urinary tract. Compr Physiol 5:327-396.

Domino M, Pawlinski B, Gajewski Z (2017) Biomathematical pattern of 
EMG signal propagation in smooth muscle of the non-pregnant porcine uterus. PloS One 12:e0173452.

Filli L, Engmann AK, Zörner B, Weinmann O, Moraitis T, Gullo M, Kasper H, Schneider R, Schwab ME (2014) Bridging the gap: a reticulopropriospinal detour bypassing an incomplete spinal cord injury. J Neurosci 34:13399-13410.

Foditsch EE, Roider K, Sartori AM, Kessler TM, Kayastha SR, Aigner L, Schneider MP (2018) Cystometric and external urethral sphincter measurements in awake rats with implanted catheter and electrodes allowing for repeated measurements. J Vis Exp. Advance online publication. Retrieved January 30, 2018. doi: 10.3791/56506.

Fowler CJ, Griffiths D, de Groat WC (2008) The neural control of micturition. Nat Rev Neurosci 9:453-466.

Freund P, Schmidlin E, Wannier T, Bloch J, Mir A, Schwab ME, Rouiller EM (2006) Nogo-A-specific antibody treatment enhances sprouting and functional recovery after cervical lesion in adult primates. Nat Med 12:790-792.

Griffiths D, Holstege G, Dalm E, Wall HD (1990) Control and coordination of bladder and urethral function in the brainstem of the cat. Neurourol Urodyn 9:63-82.

Groen J, Pannek J, Castro Diaz D, Del Popolo G, Gross T, Hamid R, Karsenty G, Kessler TM, Schneider M, 't Hoen L, Blok B (2016) Summary of European Association of Urology (EAU) guidelines on neuro-urology. Eur Urol 69:324-333.

Ineichen BV, Schnell L, Gullo M, Kaiser J, Schneider MP, Mosberger AC, Good N, Linnebank M, Schwab ME (2017) Direct, long-term intrathecal application of therapeutics to the rodent CNS. Nat Protoc 12:104-131.

Keller JA, Chen J, Simpson S, Wang EH, Lilascharoen V, George O, Lim BK, Stowers L (2018) Voluntary urination control by brainstem neurons that relax the urethral sphincter. Nat Neurosci 21:1229-1238.

Kim EH, Ryu DH, Hwang S (2011) The expression of corticotropinreleasing factor and its receptors in the spinal cord and dorsal root ganglion in a rat model of neuropathic pain. Anat Cell Biol 44:60-68.

Kucher K, Johns D, Maier D, Abel R, Badke A, Baron H, Thietje R, Casha S, Meindl R, Gomez-Mancilla B, Pfister C, Rupp R, Weidner N, Mir A, Schwab ME, Curt A (2018) First-in-man intrathecal application of neurite growth-promoting anti-Nogo-A antibodies in acute spinal cord injury. Neurorehabil Neural Repair 32:578-589.

Liebscher T, Schnell L, Schnell D, Scholl J, Schneider R, Gullo M, Fouad K, Mir A, Rausch M, Kindler D, Hamers FP, Schwab ME (2005) Nogo-A antibody improves regeneration and locomotion of spinal cord-injured rats. Ann Neurol 58:706-719.

Lindau NT, Bänninger BJ, Gullo M, Good NA, Bachmann LC, Starkey ML, Schwab ME (2014) Rewiring of the corticospinal tract in the adult rat after unilateral stroke and anti-Nogo-A therapy. Brain 137:739-756.

Marson L (1997) Identification of central nervous system neurons that innervate the bladder body, bladder base, or external urethral sphincter of female rats: a transneuronal tracing study using pseudorabies virus. J Comp Neurol 389:584-602.

Miyazato M, Sugaya K, Nishijima S, Ashitomi K, Hatano T, Ogawa Y (2003) Inhibitory effect of intrathecal glycine on the micturition reflex in normal and spinal cord injury rats. Exp Neurol 183:232-240.

Miyazato M, Sugaya K, Nishijima S, Ashitomi K, Morozumi M, Ogawa Y (2005) Dietary glycine inhibits bladder activity in normal rats and rats with spinal cord injury. J Urol 173:314-317.

Miyazato M, Sasatomi K, Hiragata S, Sugaya K, Chancellor MB, de Groat WC, Yoshimura N (2008a) GABA receptor activation in the lumbosacral spinal cord decreases detrusor overactivity in spinal cord injured rats. J Urol 179:1178-1183.

Miyazato M, Sasatomi K, Hiragata S, Sugaya K, Chancellor MB, de Groat WC, Yoshimura N (2008b) Suppression of detrusor-sphincter dyssynergia by GABA-receptor activation in the lumbosacral spinal cord in spinal cord-injured rats. Am J Physiol Regul Integr Comp Physiol 295:R336R342.
Müllner A, Gonzenbach RR, Weinmann O, Schnell L, Liebscher T, Schwab ME (2008) Lamina-specific restoration of serotonergic projections after Nogo-A antibody treatment of spinal cord injury in rats. Eur J Neurosci 27:326-333.

Nadelhaft I, Booth AM (1984) The location and morphology of preganglionic neurons and the distribution of visceral afferents from the rat pelvic nerve: a horseradish peroxidase study. J Comp Neurol 226:238-245.

Nadelhaft I, Vera PL (2001) Separate urinary bladder and external urethral sphincter neurons in the central nervous system of the rat: simultaneous labeling with two immunohistochemically distinguishable pseudorabies viruses. Brain Res 903:33-44.

Nosseir M, Hinkel A, Pannek J (2007) Clinical usefulness of urodynamic assessment for maintenance of bladder function in patients with spinal cord injury. Neurourol Urodyn 26:228-233.

Oertle T, van der Haar ME, Bandtlow CE, Robeva A, Burfeind P, Buss A, Huber AB, Simonen M, Schnell L, BrösamleC, Kaupmann K, Vallon R, Schwab ME (2003) Nogo-A inhibits neurite outgrowth and cell spreading with three discrete regions. J Neurosci 23:5393-5406.

Panicker JN, Fowler CJ, Kessler TM (2015) Lower urinary tract dysfunction in the neurological patient: clinical assessment and management. Lancet Neurol 14:720-732.

Schneider MP, Hughes FM Jr, Engmann AK, Purves JT, Kasper H, Tedaldi M, Spruill LS, Gullo M, Schwab ME, Kessler TM (2015) A novel urodynamic model for lower urinary tract assessment in awake rats. BJU Int 115 [Suppl 6]:8-15.

Schöps TF, Schneider MP, Steffen F, Ineichen BV, Mehnert U, Kessler TM (2015) Neurogenic lower urinary tract dysfunction (NLUTD) in patients with spinal cord injury: long-term urodynamic findings. BJU Int 115 [Suppl 6]:33-38.

Schwab ME (2010) Functions of Nogo proteins and their receptors in the nervous system. Nat Rev Neurosci 11:799-811.

Schwab ME, Strittmatter SM (2014) Nogo limits neural plasticity and recovery from injury. Curr Opin Neurobiol 27:53-60.

Sie JA, Blok BF, de Weerd H, Holstege G (2001) Ultrastructural evidence for direct projections from the pontine micturition center to glycineimmunoreactive neurons in the sacral dorsal gray commissure in the cat. J Comp Neurol 429:631-637.

Simpson LA, Eng JJ, Hsieh JT, Wolfe DL, Wolfe DL (2012) The health and life priorities of individuals with spinal cord injury: a systematic review. J Neurotrauma 29:1548-1555.

Skofitsch G, Zamir N, Helke CJ, Savitt JM, Jacobowitz DM (1985) Corticotropin releasing factor-like immunoreactivity in sensory ganglia and capsaicin sensitive neurons of the rat central nervous system: colocalization with other neuropeptides. Peptides 6:307-318.

Solomonow M, Baten C, Smit J, Baratta R, Hermens H, D’Ambrosia R, Shoji H (1990) Electromyogram power spectra frequencies associated with motor unit recruitment strategies. J Appl Physiol 68:1177-1185.

Studeny S, Vizzard MA (2005) Corticotropin-releasing factor (CRF) expression in postnatal and adult rat sacral parasympathetic nucleus (SPN). Cell Tissue Res 322:339-352.

Valentino RJ, Page ME, Luppi PH, Zhu Y, Van Bockstaele E, Aston-Jones G (1994) Evidence for widespread afferents to Barrington's nucleus, a brainstem region rich in corticotropin-releasing hormone neurons. Neuroscience 62:125-143.

Verstegen AMJ, Vanderhorst V, Gray PA, Zeidel ML, Geerling JC (2017) Barrington's nucleus: neuroanatomic landscape of the mouse "pontine micturition center". J Comp Neurol 525:2287-2309.

Wahl AS, Omlor W, Rubio JC, Chen JL, Zheng H, Schröter A, Gullo M, Weinmann O, Kobayashi K, Helmchen F, Ommer B, Schwab ME (2014) Asynchronous therapy restores motor control by rewiring of the rat corticospinal tract after stroke. Science 344:1250-1255.

Weld KJ, Graney MJ, Dmochowski RR (2000) Clinical significance of detrusor sphincter dyssynergia type in patients with post-traumatic spinal cord injury. Urology 56:565-568. 